\title{
Transection of Sternocleidomastoid for Selective Neck Dissection in Recurrent Papillary Thyroid Cancers
}

\author{
${ }^{1}$ Sabaretnam Mayilvaganan, ${ }^{2}$ Sapana Bothra, ${ }^{3}$ Mohammed Rashid, ${ }^{4}$ Aromal Chekavar \\ ${ }^{5}$ Ashok Kumar Vema, ${ }^{6}$ Amit Agarwal
}

\begin{abstract}
The surgical treatment of papillary thyroid carcinoma is the most frequent procedure in endocrine oncologic practice for endocrine surgeons. With the advent of positron emission tomography scan, many of these patients who had previous surgery in the form of total thyroidectomy with neck dissection present with recurrent nodal metastasis which is the cause of thyroglobulin increase. Reoperative surgery is fraught with increased incidence of complications mainly the internal jugular vein injury and nerve injuries. In this scenario, good exposure helps in avoiding these injuries, and also helps the surgeon. We describe the exposure of the posterior triangle by the division of sternocleidomastoid superiorly off the mastoid tip and retracting the muscle downward and suturing it back at the end of the procedure.
\end{abstract}

Keywords: Neck Dissection, Sternocleidomastoid, Total thyroidectomy.

How to cite this article: Mayilvaganan S, Bothra S, Rashid M, Chekavar A, Vema AK, Agarwal A. Transection of Sternocleidomastoid for Selective Neck Dissection in Recurrent Papillary Thyroid Cancers. World J Endoc Surg 2017;9(1):32-34.

Source of support: Nil

Conflict of interest: None

\section{INTRODUCTION}

The surgical treatment of papillary thyroid carcinoma is by far the most frequent procedure in endocrine oncologic practice. With the advent of positron emission tomography scan, many of these patients who had previous surgery in the form of total thyroidectomy with central compartment neck node dissection with selective neck node dissection of the lateral compartment (usually IIB, III,IV, VA) present with nodal metastasis which is the cause of thyroglobulin increase and no scan detected disease. Reoperative surgery is fraught with increased incidence of complications

\footnotetext{
${ }^{1}$ Assistant Professor, ${ }^{2-4}$ Senior Resident, ${ }^{5-6}$ Professor

${ }^{1-6}$ Department of Endocrine and Breast Surgery, Sanjay Gandhi Postgraduate Institute of Medical Sciences, Lucknow, Uttar Pradesh, India

Corresponding Author: Amit Agarwal, Professor, Department of Endocrine and Breast Surgery, Sanjay Gandhi Postgraduate Institute of Medical Sciences, Lucknow, Uttar Pradesh, India Phone: +918004904645, e-mail: amit@sgpgi.ac.in
}

mainly the internal jugular vein injury and nerve injuries. A thorough knowledge of neck anatomy and understanding the extent of the disease is key to success of surgery with minimal complication. The understanding of basic systematic surgical approach may result in an optimal exposure of the neck nodes and decreases the complications. We describe the exposure of posterior triangle by division of sternocleidomastoid (SCM) superiorly off the mastoid tip. This procedure of transection of SCM at its insertion provides a gateway to enter posterior triangle safely, especially in a reoperative surgery with patient already undergone lymph node dissection. ${ }^{1}$

\section{STERNOCLEIDOMASTOID MUSCLE}

The SCM is a two headed muscle. It is innervated by cranial accessory nerve and direct branches of the cervical plexus C1-C2. Sternal head originates from the manubrium sterni. Clavicular head originates from the middle part of the clavicle. The insertion is into the mastoid process of temporal bone and superior nuchal line of the occipital bone (Fig. 1).

\section{FUNCTION}

A unilateral contraction of the SCM flexes the cervical vertebral column to the same side (lateral flexion) and rotates the head to the opposite side. A bilateral innervation elevates the head by dorsally extending the upper

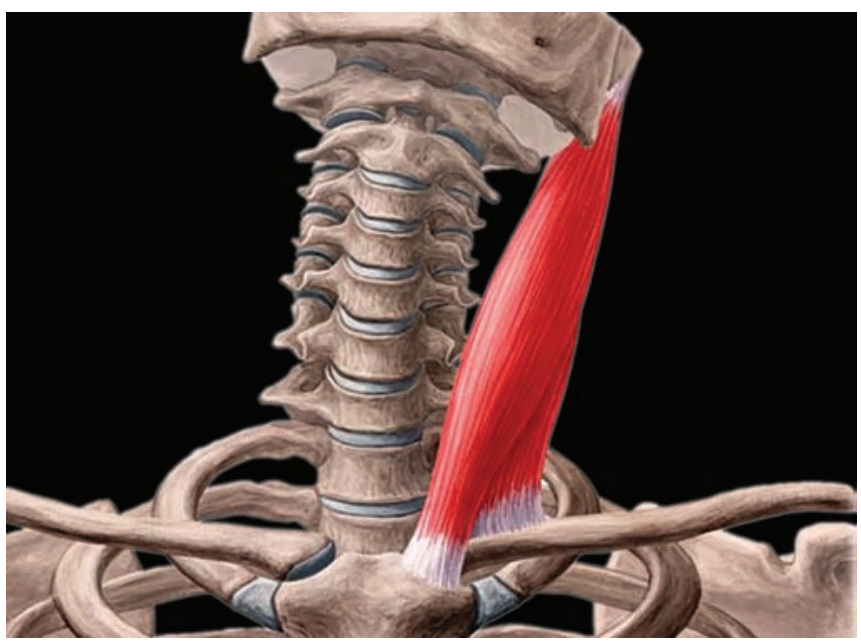

Fig. 1: Origin and Insertion of sternocleidomastoid muscle 


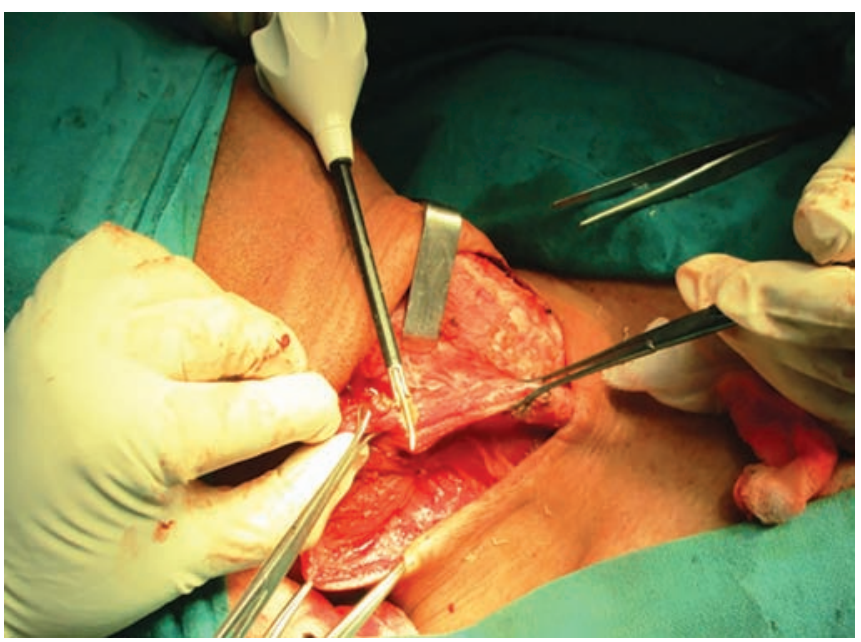

Fig. 2: Sternocleidomastoid transected at the insertion

cervical joints. If the head is fixed, it elevates the sternum and clavide and thus expands the thoracic cavity (muscle of inspiration).

Blood supply can be divided into three parts: Upper, middle, and lower. The upper third supplied by occipital artery. The middle third receives a branch from super thyroidectomy artery (42\%), external carotid artery (27\%), or both $(23 \%)$. The lower third of the muscle is supplied by suprascapular artery. 2,3

\section{Variations}

The clavicular origin of SCM varies greatly; in some cases the clavicular head may be as narrow as the sternal and in others, it may be as much as $7.5 \mathrm{~cm}$ in breadth. When the clavicular origin is broad, it is occasionally subdivided into several slips separated by narrow intervals. More rarely the adjoining margins of the SCM and trapezius are in contact. This would leave no posterior triangle. The supraclavicularis muscle arises from the manubrium behind SCM and passes behind the SCM to the upper surface of the clavicle.

\section{Transection of Sternocleidomastoid}

The SCM muscle is mobilized from the surrounding structures. The medical and lateral margins are mobilized. To mobilize the margins, the muscle is held after mobilization in a single place using a gauze piece tied and the entire muscle pulled-up. This opens up a good plane of dissection. Once the entire muscle is mobilized, care is taken to cut it just below the insertion into the mastoid process (Fig. 2). This shall be done using electrocautery or ultrasonic energy source. The cut end is then retracted downward which helps in the entry to level 5 and also dissection of highly placed level 2 nodes (Fig. 3).

The nodes which are medial to the SCM, if any, should be dissected before thismuscle mobilization maneuver, because the maintenance of muscle bar results in a

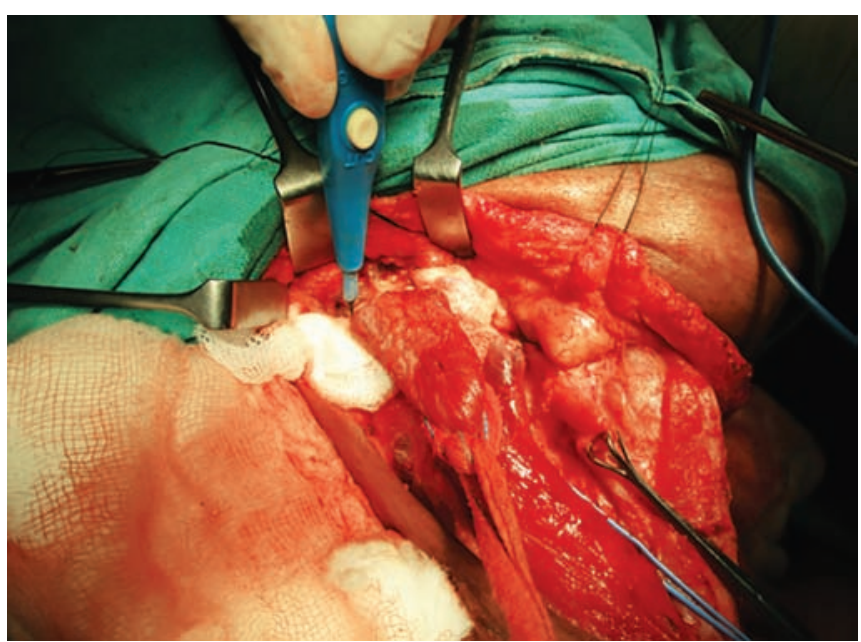

Fig. 3: Mastoid process in view and muscle belly retracted downward

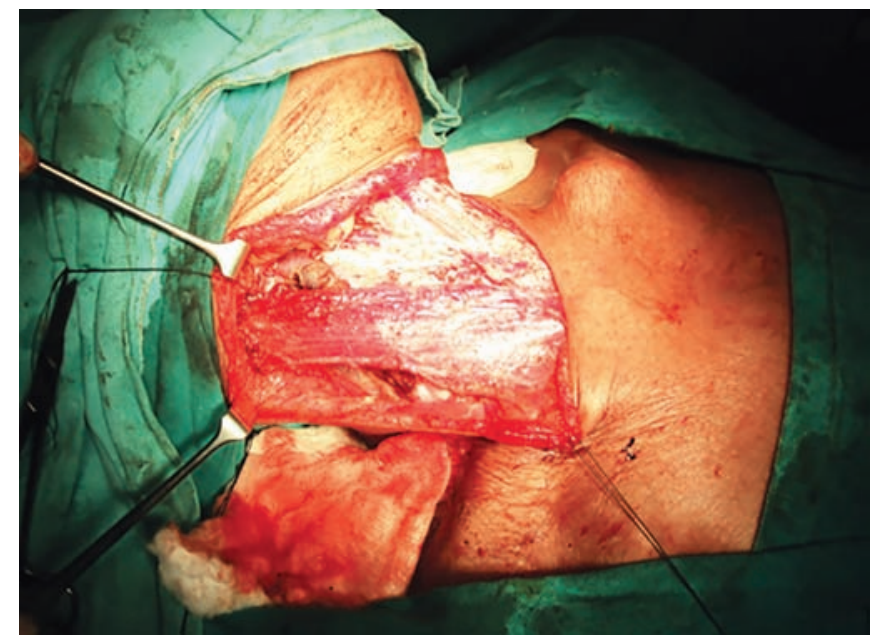

Fig. 4: Sutured SCM at the end of surgery

preserved crania-caudal tension in the neck dissection specimen. Under the sternocleido mastoid, a thick layer of adipose tissue is encountered which can contain transverse cervical artery and vein and major or minor lymphatic duets with variable convections to internal jugular vein. Dissection should therefore be performed with great care.

This transaction of SCM high-up preserves special accessory nerve since the most constant source of blood supply is to the lower third of SCM and cutting it high-up can result in preservation of blood supply and post of functionality (Fig. 4). ${ }^{4}$

\section{CONCLUSION}

Authors have found this technique quite useful for reoperative surgeries especially for highly placed level 2 nodes where exposure is quite important and if IJV is injured, it may be a disaster. The preservation of SCM results in preservation of the neck contour and also covers the internal jugular vein and carotid artery. The cosmetic acceptability is also good. 


\section{REFERENCES}

1. Balm AJ, Lohuis PJ, Copper MP. Surgical techniqueunwrapping the neck node levels around a sternocleidomastoid muscle bar: a systematic way of performing (modified) radical neck dissection. Eur J Surg Oncol 2005 Dec;31(10): 1216-1221.

2. Leclère FM, Vacher C, Benchaa T. Blood supply to the human sternocleidomastoid muscle and its clinical implications for mandible reconstruction. Laryngoscope 2012 Nov;122(11): 2402-2406.

3. Kierner AC, Aigner M, Zelenka I, Riedl G, Burian M. The blood supply of the sternocleidomastoid muscle and its clinical implications. Arch Surg. 1999 Feb;134(2):144-147.

4. Cuccia G, Shelley OP, d'Alcontres FS, Giannitrapani M, Soutar DS, Camilleri IG. Evidence of significant sternocleidomastoid atrophy following modified radical neck dissection type III. Plast Reconstr Surg. 2006 Jan;117(1):227-232. 